\title{
The Real-Life Effectiveness and Care Patterns of Diabetes Management Study for Balkan Region (Slovenia, Croatia, Serbia, Bulgaria): A Multicenter, Observational, Cross-Sectional Study
}

\author{
Sanja Klobucar Majanovic (D) - Andrej Janez - Ivaylo Lefterov • \\ Sanja Tasic $\cdot$ Tatjana Cikac \\ Received: May 1, 2017 / Published online: July 10, 2017 \\ (C) The Author(s) 2017. This article is an open access publication
}

\begin{abstract}
Introduction: Recent large randomized controlled trials highlighted the clinical significance of hypoglycemic episodes in the treatment of diabetes. The present survey was conducted to provide information from real-life practice on the incidence of hypoglycemia in type 2 diabetic patients treated with sulfonylureas.
\end{abstract}

Enhanced content To view enhanced content for this article go to http://www.medengine.com/Redeem/ 10A8F0604C1630D0.

Electronic supplementary material The online version of this article (doi:10.1007/s13300-017-0288-x) contains supplementary material, which is available to authorized users.

S. K. Majanovic $(\square)$

Division of Endocrinology, Diabetes and Metabolic

Diseases, Department of Internal Medicine, University Hospital Rijeka, Rijeka, Croatia

e-mail: sanja.klobucar@medri.uniri.hr

A. Janez

Department of Endocrinology, Diabetes and Metabolic Diseases, University Medical Centre

Ljubljana, Ljubljana, Slovenia

I. Lefterov

MHAT 5 Sofia EAD, Sofia, Bulgaria

S. Tasic

Novi Beograd Health Center, Belgrade, Serbia

T. Cikac

Family General Practice, Petra Preradovica 25,

Varazdin, Croatia
Methods: This multicenter, observational, cross-sectional study collected data on incidence of side effects of sulfonylurea-based therapy in type 2 diabetic patients in four countries of the Balkan region (Slovenia, Croatia, Serbia, Bulgaria) from October 2014 to June 2015.

Results: Of the 608 who participated in the study, 573 patients (mean age 67.2 years, mean body mass index $29.9 \mathrm{~kg} / \mathrm{m}^{2}$ ) met the inclusion/ exclusion criteria. More than $90 \%$ of the patients were treated with the newer generation sulfonylureas-gliclazide or glimepiride-either as monotherapy or as dual therapy in combination with metformin. In total, 210 patients (36.6\%) reported hypoglycemic episode(s) in the last 6 months. Mild episodes were reported by 132 patients $(62.8 \%)$, moderate by 66 (31.2\%), severe by 8 patients $(4.0 \%)$, and very severe by 4 patients (2\%), respectively. Overall, 171 patients $(28.2 \%)$ reported body weight increase during the previous year. The mean reported body weight gain in this group of patients was $4.2 \mathrm{~kg}$ (SD 2.7, median 3). Among them, $68.1 \%$ gained less than $5 \mathrm{~kg}, 25.0 \%$ gained $5-9 \mathrm{~kg}$, and the rest gained more than $10 \mathrm{~kg}$.

Conclusion: Although newer generation sulfonylureas are generally considered safe in terms of hypoglycemia, our data indicates their use is associated with substantial risk of hypoglycemia and weight gain. Clinicians should be mindful of these findings when prescribing SUs 
and inform patients about the risk of hypoglycemia.

Funding: Merck Sharp \& Dohme.

Keywords: Fear of hypoglycemia Hypoglycemia; Real-life diabetes management; Sulfonylureas

\section{INTRODUCTION}

Although the medical benefits of improved glycemic control have gained wide recognition, diabetes management aimed toward increasingly stringent glycemic goals may be associated with hypoglycemia. Hypoglycemia is the most common and highly feared acute complication of diabetes treatment that may adversely affect clinical outcomes and quality of life [1]. Recent findings from large randomized controlled trials (RCTs) highlighted the clinical significance of hypoglycemic episodes and led to an increased emphasis on the latter in international treatment guidelines [2-4]. Several trials demonstrated that intensive glycemic control was associated with an increased incidence of hypoglycemia and even suggested hypoglycemia to be associated with excess mortality [5-7]. It is well known that the risk of hypoglycemia increases with advanced age, duration of diabetes, and the presence of severe comorbidities and hypoglycemia unawareness [8]. Insulin and sulfonylureas (SUs) are the agents that most commonly cause hypoglycemia [9]. Although the risk of hypoglycemia is particularly elevated in patients receiving insulin therapy, patients with type 2 diabetes mellitus (T2DM) treated with insulin secretagogues (e.g., sulfonylureas, meglitinides) are also at increased risk of experiencing hypoglycemia [10]. The well-being of patients with T2DM may be affected both directly as a result of hypoglycemic symptoms and indirectly due to fear of recurring hypoglycemia [11]. Moreover, even mild hypoglycemic episodes may be enough to adversely impact clinical management of diabetes if patients' fear compromises their willingness to take medications as prescribed. Awareness of these factors are reflected in amendments of international guideline recommendations (ADA/EASD) [12]. However, implementation of recommended strategies requires relevant information from real-life practice to be available. There is a need to investigate the clinical spectrum and burden of hypoglycemia so that adequate preventive measures can be implemented against this life-threatening complication. This was the rationale for the present observational, cross-sectional study designed to assess the incidence rate of side effects of SU therapy such as hypoglycemia, evaluated by patient-reported outcomes in countries of the Balkan region. The survey also addressed some other (health- and/ or quality of life (QoL)-related) aspects, including body weight gain and evaluation of how much patients are bothered by these side effects.

\section{METHODS}

\section{Study Design}

The Real-Life Effectiveness and Care Patterns of Diabetes Management (RECAP-DM) Balkan study is an observational, multicenter, cross-sectional study including patients with T2DM on SU therapy. The study was conducted in primary care physician practices and diabetology/endocrinology outpatient clinics in Slovenia, Croatia, Serbia, and Bulgaria. Study centers were randomly selected among a convenience sample of physicians from each of the four countries. The target was to recruit a total of 10-20 physicians in each participating country. The primary objectives were to assess the incidence rate of side effects of SU therapy such as hypoglycemia, evaluated by patient-reported outcomes and weight gain, as well as by how much patients were bothered by these side effects. Another primary objective was to assess the adequacy of glycemic control. Adequate glycemic control (at HbA1c target) was defined according to the International Diabetes Federation (IDF) as HbA1c less than 6.5\%, and according to the American Diabetes Association (ADA) as HbA1c less than $7.0 \%$. The secondary objectives were to describe the demographic and medical factors associated with different levels of hypoglycemia and glycemic control. 


\section{Sample Size Calculation}

The prevalence of hypoglycemia was reported at $35.8 \% \quad(95 \%$ CI $33.8-37.8 \%)$ in the AP RECAP-DM study [13], and at $38.4 \%$ (95\% CI $34.0-42.8 \%$ ) in the EU RECAP-DM study. Country-specific prevalence ranged from $24.2 \%$ (Germany) to $53.6 \%$ (UK) [14]. As this interval includes the "worst case scenario" proportion of $50 \%$, it was used as a base for power calculations. Assuming a confidence level of $95 \%$ and prevalence of $50 \%, 153$ patients per country were required to ensure the desired error margin of $\pm 10 \%$ with a power of 0.8 . The study was sized according to the primary objectives (establishing prevalence of hypoglycemia and poor disease control in the region).

Finally, a total of 57 physicians participated in the study (Bulgaria 6 sites/15 HCP—GPs/149 patients/ca. 10 patients each; Croatia 13 sites/13 HCP-GPs/144 patients/ca. 10 patients each, some 12; Serbia 14 sites/14 HCP-GPs/153 patients/ca. 9 patients each, some up to 18; Slovenia 14 sites/15 HCP-only diabetologists due to treatment paradigm that only specialists treat diabetes/161 patients/ca. 10 patients each, some 13).

\section{Inclusion/Exclusion Criteria}

Eligible patients according to the inclusion/exclusion criteria listed in Table 1 were enrolled in the study from October 2014 to June 2015. To reduce selection bias, physicians were asked to enroll 10-15 eligible patients consecutively. All patients signed an informed consent form prior to enrollment. Both the study protocol and informed consent form were approved by the local ethical committee in each country.

Case report forms were used to collect data on patient demographic and clinical characteristics from medical records during the 6-month period prior to the enrollment date. These included patient age and gender, duration of diabetes, age at diagnosis of diabetes, smoking status, alcohol use, weight, body mass index, physical activity and antidiabetic therapy prior to the visit date, as well as therapy
Table 1 Inclusion/exclusion criteria

Inclusion criteria
Patient diagnosed with type 2 diabetes mellitus (DM)
(ADA criteria)
$\geq 30$ years at the time of type $2 \mathrm{DM}$ diagnosis
Treatment with SU (as monotherapy or in
combination with metformin) for at least 6 months
prior to enrollment (as evidenced by filled
prescriptions for at least 90 -day supply)
Patient has his/her clinical record in the healthcare
center
Patient's medical records include the minimum core
data
Patient is primarily managed in the healthcare center
Patient properly completes the questionnaires (after
having given consent)
Outpatient patient

\section{Exclusion criteria}

Patients with type $1 \mathrm{DM}$

Pregnant women/women with gestational DM

Patients who have been taking DPP-4 inhibitors, GLP-1 receptor agonists, meglitinides, pioglitazone, or insulin during the 6 months prior to enrollment

Patients who are unable to complete the questionnaire for whatever reason

Patients who are already participating in another clinical trial

Inability or unwillingness to sign the informed consent

Investigator's decision that the patient should not take part in the study

modifications at the study visit. Data on glycemic control were based on the most recent HbA1c measurement within a month prior to the visit date. Otherwise, patients were subjected to a standard blood draw during the physician office visit (i.e., patient enrollment date) to obtain HbA1c measurement. 


\section{Patient-Reported Outcomes}

At study visit patients were administered a study-specific questionnaires (see Online Appendix) consisting of questions/items on general adherence, general self-care, experience of hypoglycemia, weight gain and fear of weight gain, and the standard Hypoglycemia Fear Survey II Worry Subscale (HFS-II WS) on fear of hypoglycemia.

According to ADA guidelines hypoglycemic events are classified as severe hypoglycemia, documented symptomatic hypoglycemia, asymptomatic hypoglycemia, probable symptomatic hypoglycemia, or pseudo-hypoglycemia [12]. As a result of the real-world setting and self-reported outcome measures in the study design, we decided to use an approach that was more convenient for the patients. Therefore, severity of hypoglycemia was classified as (1) mild (no or little interruption of activities and no assistance required to manage symptoms); (2) moderate (some interruption of activities and no assistance needed to manage symptoms); (3) severe (requiring third-party assistance to manage symptoms); and (4) very severe (hypoglycemia episode that required medical assistance). Patients were asked to read a list of hypoglycemic symptoms and quantify the frequency and severity of such symptoms during the 6 months preceding the survey. If a patient reported episodes of hypoglycemia at more than one level of severity, the participant was categorized according to the most severe level reported. The frequency data were collected as 1-2 episodes over the last 6 months, 3-6 episodes over the last 6 months, more than one per month, more than one per week, or daily.

Worry about hypoglycemia was measured using the HFS-II WS. The administered subscale consists of 18 items that measure degree of patient fear in the past 6 months with responses ranging from 0 to 4 points (higher value denoting more pronounced worries), with possible total scores from 0 to 72 points.

Evaluation of weight gain was based on patients' self-reported unintentional body weight increase during the last year. The questionnaire administered to patients at the study visit included questions about weight gain (five items) and about fear of weight gain (three items) (see Online Appendix).

\section{Compliance with Ethics Guidelines}

All procedures followed were in accordance with the ethical standards of the responsible committee on human experimentation (institutional and national) and with the Helsinki Declaration of 1964, as revised in 2013. Informed consent was obtained from all patients for being included in the study.

\section{Statistical Analyses}

All statistical analysis was performed using Statistical Package for the Social Sciences (SPSS) version 17.0 for Windows (SPSS, Chicago, IL). Descriptive statistics was used to summarize patient demographics, disease characteristics, medication side effects, and patient-reported outcomes. Post hoc pairwise Chi-squared test with Bonferroni correction was used to test the differences between proportions. Mann-Whitney test was used for data that are not normally distributed. A $p$ value of 0.05 was considered statistically significant. A multivariate approach using logistic regression was employed to explore association of glycemic control and hypoglycemic episodes with patient characteristics. The included continuous variables (age, duration of diabetes) were intentionally not dichotomized/categorized. Given the exploratory nature of the model, only main effects (without interactions) were fitted to keep it simple. In addition, exploratory univariate analyses were performed with variables of interest not included in the logistic regression models (using Mann-Whitney or Fisher test, as appropriate, with Bonferroni correction).

\section{RESULTS}

\section{Patient Characteristics}

Of the 608 who participated in the study, after the database lock it was found that 34 patients 
Table 2 Patient characteristics

\begin{tabular}{lc}
\hline Characteristics & Mean $(\mathrm{SD}) / \boldsymbol{n}(\%)$ \\
\hline Age (years) & $67.2(9.7)$ \\
$\geq 65$ years & $364(63.5 \%)$ \\
Gender & \\
Female & $287(50.1 \%)$ \\
Male & $286(49.9 \%)$ \\
HbAlc $(\%)$ & $7.1(1.4)$ \\
HbAlc at target $<7.0 \%$ & $316(55.1 \%)$ \\
HbAlc at target $<6.5 \%$ & $193(33.7 \%)$ \\
Weight $(\mathrm{kg})$ & $84.5(16.6)$ \\
Body mass index $\left(\mathrm{kg} / \mathrm{m}^{2}\right)$ & $29.9(5.2)$ \\
Duration of diabetes $($ years $)$ & $9.9(6.4)$ \\
Age at diagnosis $($ years $)$ & $57.4(9.8)$ \\
Current smokers & $65(11.3)$ \\
\hline
\end{tabular}

did not fulfill the inclusion or exclusion criteria, and one patient record was found to contain 90 missing data (out of 96). All these 35 patients were omitted from the final analysis. For the remaining 573 patients, the mean age was 67.2 years, $63.5 \%$ of all patients were at least 65 years of age, $50.1 \%$ were female, and the mean body mass index was $29.9 \mathrm{~kg} / \mathrm{m}^{2}$. The average duration of diabetes was 9.9 years, and $80.8 \%$ of patients had diabetes for at least 5 years. The mean HbA1c level was $7.1 \%$, and only $33.7 \%$ of patients were at HbA1c goal according to IDF criteria (HbA1c less than 6.5\%) and $55.1 \%$ according to ADA criteria (HbA1c less than $7.0 \%$ ), respectively (Table 2 ).

\section{Antidiabetic Therapy}

Up to the study visit 161 (28.1\%) patients were treated with SU as monotherapy and 412 (71.9\%) were taking dual therapy (metformin $+\mathrm{SU}$, except two patients receiving SU and acarbose). The SUs administered were glibenclamide, gliclazide, glimepiride, glipizide, or gliquidone. The number/proportion of individual active substances among patients on monotherapy and dual therapy are shown in
Table 3 The number/proportion of individual active substances among patients on monotherapy and dual therapy

\begin{tabular}{lcc}
\hline & $\begin{array}{l}\text { Number } \\
\text { of patients } \\
(\boldsymbol{n})\end{array}$ & $\begin{array}{l}\text { Proportion } \\
\text { of patients } \\
(\%)\end{array}$ \\
\hline Monotherapy & 91 & 56.5 \\
Gliclazide & 53 & 32.9 \\
Glimepiride & 9 & 5.6 \\
Gliquidone & 5 & 3.1 \\
Glipizide & 3 & 1.9 \\
Glibenclamide & 189 & 45.9 \\
Dual therapy & 186 & 45.1 \\
Gliclazide + metformin & 18 & 4.4 \\
Glimepiride + metformin & 12 & 2.9 \\
Gliquidone + metformin & 5 & 1.2 \\
Glipizide + metformin & 12 & 0.5 \\
\hline Glibenclamide + metformin & 5 & \\
Gliclazide + acarbose & 2 & \\
\hline
\end{tabular}

Table 3. The average doses of individual substances were as follows: gliclazide $56.5 \mathrm{mg}$, glimepiride $2.4 \mathrm{mg}$, gliquidone $30 \mathrm{mg}$, glipizide $7.4 \mathrm{mg}$, glibenclamide $3.9 \mathrm{mg}$, metformin $857.3 \mathrm{mg}$, and acarbose $50 \mathrm{mg}$. At the study visit therapy was changed in 160 patients (28\%). The majority of modifications consisted of dosage adjustments (88 patients) or change of diabetic treatment in the same pharmacological class (12 patients). Addition/switch to another pharmacological class was reported in only 60 patients (10.5\% of study population). Overall, introduction of a DPP-4 inhibitor was the most prevalent change when adding/switching to a different pharmacological class; specifically, 17 patients received a DPP-4 inhibitor (sitagliptin 14, linagliptin 2, unspecified 1).

\section{Hypoglycemic Episodes}

In total, 210 patients (36.6\%) reported hypoglycemic episode(s) in the last 6 months. Mild episodes were reported by 132 patients $(62.8 \%)$, 
Table 4 Experience of hypoglycemia

\begin{tabular}{ll}
\hline Occurrence of hypoglycemic episodes & \% of cases \\
\hline 1-2 times over the last 6 months & 40.1 \\
3-6 times over the last 6 months & 28.8 \\
More than once per month & 17.5 \\
More than once per week & 10.3 \\
Every day & 3.3 \\
\hline
\end{tabular}

moderate by $66(31.2 \%)$, severe by 8 patients $(4.0 \%)$, and very severe by 4 patients $(2 \%)$, respectively. Overall episodes of hypoglycemia occured as shown in Table 4.

\section{HFS-II Worry Subscale Score}

Additional (indirect) assessment of burden of hypoglycemia was performed using the Worry Subscale of the HFS-II questionnaire. Relative proportions of responses to HFS-II Worry Subscale items are presented in Table 5. Mean HFS-II Worry Subscale score was 8.8 (SD 11.5, median 4 , range $0-72$ ). There was a statistically significant difference in the HFS scores between patients with and without hypoglycemic episodes (estimated median difference 4 points, 95\% CI 2-5, $p<0.0001$; Mann-Whitney test). HFS scores were also compared within the subgroup of patients reporting hypoglycemic episodes with respect to the severity of episodes (defined as the most severe episode experienced). As it was not possible to analyze very severe cases as a separate group (because of their small number), they were merged with severe cases. Statistically significant HFS score differences were found between patients with mild vs. severe episodes and between those with moderate vs. severe episodes (both $p<0.0001$; Tukey HSD test), while difference between patients with mild vs. moderate episodes was not significant (Fig. 1).

\section{Weight Gain}

Evaluation of weight gain was based on patients' self-reported body weight increase
Table 5 Relative proportions of responses to HFS-II Worry Subscale items

\begin{tabular}{|c|c|c|c|c|c|c|}
\hline \multirow[t]{2}{*}{ Item } & \multicolumn{6}{|c|}{ Relative proportions of responses (\%) } \\
\hline & Never & Rarely & Sometimes & Often & Very often & NA \\
\hline 1 & 58.8 & 22.3 & 12.7 & 4.5 & 0.3 & 1.2 \\
\hline 2 & 62.3 & 18.3 & 14.7 & 2.6 & 0.7 & 1.4 \\
\hline 3 & 65.6 & 14.3 & 15.7 & 2.1 & 0.7 & 1.6 \\
\hline 4 & 76.3 & 13.1 & 8.2 & 0.5 & 0.5 & 1.4 \\
\hline 5 & 59.3 & 17.6 & 15.5 & 4.9 & 1.2 & 1.4 \\
\hline 6 & 78.5 & 8.7 & 8.0 & 2.6 & 0.7 & 1.4 \\
\hline 7 & 71.0 & 12.2 & 12.0 & 2.3 & 0.9 & 1.6 \\
\hline 8 & 59.5 & 17.6 & 16.2 & 4.2 & 0.9 & 1.6 \\
\hline 9 & 78.9 & 8.0 & 8.0 & 1.9 & 1.0 & 2.1 \\
\hline 10 & 73.6 & 14.1 & 7.0 & 1.9 & 0.9 & 2.4 \\
\hline 11 & 81.5 & 10.1 & 4.7 & 1.0 & 1.0 & 1.6 \\
\hline 12 & 72.6 & 14.7 & 9.2 & 1.0 & 0.9 & 1.6 \\
\hline 13 & 54.3 & 21.6 & 15.9 & 5.1 & 0.7 & 2.4 \\
\hline 14 & 71.6 & 14.0 & 9.8 & 2.4 & 0.7 & 1.6 \\
\hline 15 & 64.7 & 16.8 & 12.6 & 3.0 & 1.6 & 1.4 \\
\hline 16 & 70.0 & 16.4 & 8.2 & 2.8 & 1.2 & 1.4 \\
\hline 17 & 64.6 & 14.5 & 13.8 & 4.7 & 1.0 & 1.4 \\
\hline 18 & 70.2 & 16.1 & 10.3 & 1.2 & 0.9 & 1.4 \\
\hline
\end{tabular}

Item 1 Because my blood sugar could go low, I worried about not recognizing/realizing my blood sugar was low. Item 2 Because my blood sugar could go low, I worried about not having sugary drinks or foods (such as fruit juice or candy) available to treat myself. Item 3 Because my blood sugar could go low, I worried about passing out in public. Item 4 Because my blood sugar could go low, I worried about embarrassing myself or my friends in a social or public situation. Item 5 Because my blood sugar could go low, I worried about having a hypoglycemic episode while alone. Item 6 Because my blood sugar could go low, I worried about appearing stupid or drunk to others. Item 7 Because my blood sugar could go low, I worried about losing control. Item 8 Because my blood sugar could go low, I worried about no one being around to help me during a hypoglycemic episode. Item 9 Because my blood sugar could go low, I worried about having a hypoglycemic episode while driving. Item 10 Because my blood sugar could go low, I worried about making a mistake or having an accident. Item 11 Because my blood sugar could go low, I worried about getting a bad evaluation or being criticized. Item 12 Because my blood sugar could go low, I worried about difficulty thinking clearly when responsible for others. Item 13 Because my blood sugar could go low, I worried. Item 14 Because my blood sugar could go low, I worried about accidentally injuring myself or others. Item 15 Because my blood sugar could go low, I worried about permanent injury or damage to my health or body. Item 16 Because my blood sugar could go low, I worried about low blood sugar interfering with important things I was doing. Item 17 Because my blood sugar could go low, I worried about becoming hypoglycemic during sleep. Item 18 Because my blood sugar could go low, I worried about becoming emotionally upset and difficult to deal with 


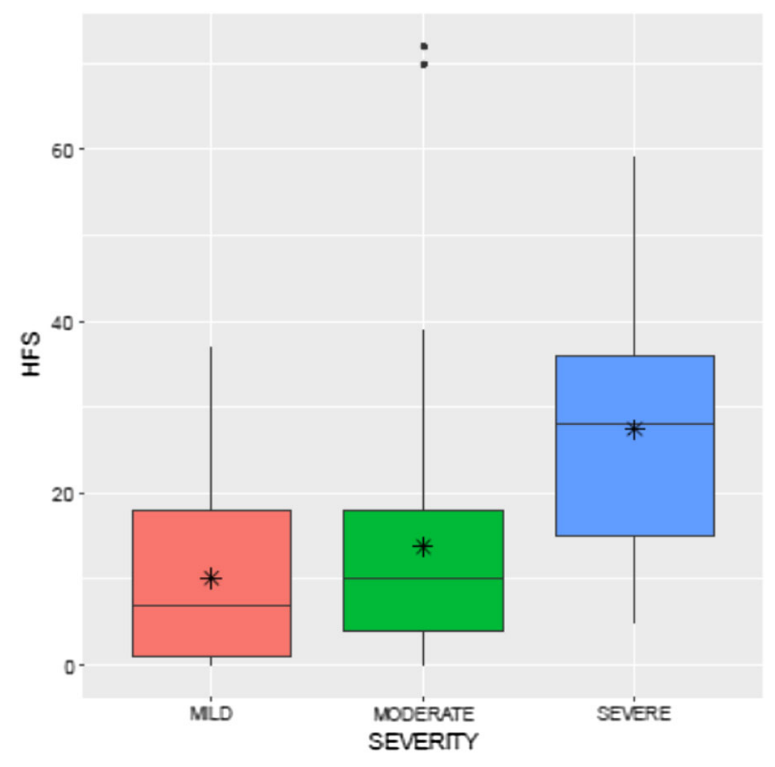

Fig. 1 Boxplot of HFS scores vs. severity of hypoglycemic episodes. Very severe cases were merged with severe cases (because of the small number). Dark horizontal lines show medians, stars within the boxes show means

during the last year. In the study sample, 171 patients $(28.2 \%)$ reported such weight gain. The mean reported body weight gain in this group of patients was $4.2 \mathrm{~kg}$ (SD 2.7, median 3). Among them, $68.1 \%$ gained less than $5 \mathrm{~kg}$, $25.0 \%$ gained $5-9 \mathrm{~kg}$, and the rest gained more than $10 \mathrm{~kg}$. There was a statistically significant trend of increasing proportion of weight gain with increasing BMI category $(p<0.0001$; Cochran-Armitage test for trend). More than $75 \%$ of patients were bothered by their weight gain. Post hoc pairwise Chi-squared test with Bonferroni correction showed significant pairwise differences for all pairs except for overweight vs. obese and obese I vs. obese II (corrected $p$ values for significant comparisons at most 0.029).

\section{Glycemic Control and Hypoglycemic Episodes in Relation to Patient Characteristics}

A multivariate approach using logistic regression was employed. However, model development was limited, partly because no model was specified in advance and partly because several potential variables of interest could not be included because of methodological issues. The first developed model associated glycemic control (yes/no according to ADA 2015 guidelines) with four variables: patient's fear of weight gain (yes/no), hypoglycemic episodes during the last 6 months (yes/no), patient age, and diabetes duration. The odds ratios (and their 95\% CIs) for the four variables were 0.681 (95\% CI 0.476 , $0.973 ; p=0.035$ ) for presence vs. absence of fear of weight gain; 1.383 (95\% CI 0.964, 1.990; $p=0.080)$ for presence vs. absence of hypoglycemic episodes in the last 6 months; 1.021 (95\% CI 1.001, 1.041; $p=0.039)$ per each additional life-year, and 0.971 (95\% CI 0.942 , $1.000 ; p=0.056)$ per each additional year of duration of diabetes. It should be noted that all four odds ratios are relatively small from the practical standpoint. The second developed model associated presence of hypoglycemic episode(s) during the last 6 months with four variables: HFS score (above/below median), gender, patient's fear of weight gain (yes/no), and glycemic control (yes/no based on ADA HbA1c criterion). The odds ratios (and their 95\% CIs) for the four variables were 3.0 (95\% CI 2.0, $4.5 ; p<0.0001)$ for HFS score above median (4 points) vs. HFS score below median; 1.54 (95\% CI 1.06, 2.25; $p=0.025)$ for female gender vs. male gender; 0.68 (95\% CI $0.45,1.059$; $p=1.00$ ) for presence vs. absence of fear of weight gain, and 1.34 (95\% CI 0.92, 2.01; $p=1.97$ ) for good glycemic control (HbA1c less than $7 \%$ ) vs. glycemic control that is not good. Of note is that this model yielded relatively high odds ratios for HFS score and gender with respect to presence of hypoglycemic episodes, indicating significantly larger odds of the latter among patients with above median HFS score vs. below median, and significantly larger odds of such episodes among women vs. men. In addition, exploratory univariate analyses were performed with variables of interest not included in the logistic regression models. The only statistically significant associations were found (1) between glycemic control and fasting plasma glucose levels (the latter being lower in well-controlled patients), which is clinically plausible; and (2) between hypoglycemic episodes and questionnaire item examining how 
bothered patients are by medication side effects, with somewhat equivocal results.

\section{DISCUSSION}

Adding sulfonylureas (SUs) to metformin still remains a commonly used strategy in the treatment of type 2 diabetes, despite well-documented safety issues related to SUs therapy such as hypoglycemia and weight gain [15]. SU treatment commonly leads to a weight gain of about $2 \mathrm{~kg}$ depending on baseline HbA1c level and two to three times higher rates of hypoglycemia than treatment with other oral antidiabetic medications [16]. A meta-analysis of 31 RCTs found that SU treatment resulted in more hypoglycemic events compared with the comparator groups (RR 2.41, 95\% CI 1.41, 4.10) but none of the studies reported any severe hypoglycemic episodes requiring third-party assistance [17]. However, as a result of the stringent restraints of RCT study designs, it is difficult to precisely determine the total burden of hypoglycemia in clinical practice. Therefore, observational studies conducted in real-world settings can provide valuable data regarding frequency and severity of hypoglycemia in the management of diabetes [18]. In the present study hypoglycemic events were reported by $36.6 \%$ of patients. The majority of patients reported mild hypoglycemia (62.8\%), about third moderate $(31.2 \%)$, and the rest severe $(4.0 \%)$ or very severe $(2.0 \%)$ hypoglycemia.

Almost $20 \%$ of our patients who reported hypoglycemic episodes experienced hypoglycemia more than once per month and 10\% more than once per week. Repeated episodes of hypoglycemia can lead to hypoglycemia unawareness, cognitive impairment, and may have a negative impact on a patient's quality of life [19]. As a result, patients can develop fear of hypoglycemia. Fear of hypoglycemia imposes an additional psychological barrier to good glycemic control and medication adherence [20]. To assess fear of hypoglycemia, researchers have most often used the Hypoglycemia Fear Survey-II (HFS-II) [21]. The HFS-II is well validated and is increasingly used in T2DM [22]. Studies that used the HFS-II score found that type 2 diabetic patients reported increased fear of hypoglycemia while the number of both mild/moderate and severe episodes increased [23]. The HFS comprises a Worry Subscale and a Behavior Subscale, with many trials using only the former (HFS-WS) [24]. HFS-WS scores are comparable between patients with type 1 diabetes and patients with type 2 diabetes receiving insulin, and scores are only moderately lower in type 2 diabetic patients on SU treatment [25]. HFS-II WS scores for our patient sample reflected the expected patterns in fear of hypoglycemia. Patients who reported hypoglycemic events showed more fear than those who did not, and patients who reported more severe episodes of hypoglycemia revealed more fear that those who reported less severe episodes. Up till now no definite cutoff score has been determined that would be most indicative of clinically relevant fear of hypoglycemia as assessed by HFS-II WS score. However, Hajós et al. suggest the use of the elevated item endorsement (EI) criterion, which supposedly shows a strong association with suboptimal patient well-being [26]. The EI criterion is fulfilled by patients who have elevated score [at least 3 (often/very often)] on more than one HFS-II WS item. Among our study participants $11.1 \%$ were found to fulfill the EI criterion.

More than $90 \%$ of the patients in our study were treated with the newer generation SUsgliclazide or glimepiride-either as monotherapy or as dual therapy in combination with metformin. According to the research findings, individual SUs differ and may confer different risks of hypoglycemia [27]. Although in contrast with the findings of previous studies, which claim that gliclazide is associated with the lowest risk of hypoglycemia among the second-generation SUs [28, 29], the results of a recently published large real-world cohort consisted of more than 100,000 participants suggest that gliclazide may not be superior to other sulfonylureas [30].

Although obesity remains one of the major risk factors for diabetes with up to $90 \%$ of diabetic patients being overweight or obese at the time of diagnosis, certain antidiabetic medications, such as insulin and SUs, can lead to substantial weight gain [31]. In our study sample 
$28.2 \%$ of patients reported body weight increase during the last year (mean $4.2 \mathrm{~kg}$, SD 2.7 , median 3). Among them, $68.1 \%$ gained less than $5 \mathrm{~kg}, 25.0 \%$ gained $5-9 \mathrm{~kg}$, and the rest gained more than $10 \mathrm{~kg}$. In addition, there was a statistically significant trend of increasing proportion of weight gain with increasing BMI category. This finding suggests that patients already endangered by their body weight tend to be at higher risk of gaining additional weight during their SU-based therapy. Patients who continue to gain weight throughout the course of their illness may enter into a vicious cycle of weight gain and poor glycemic control.

Despite significant improvement in diabetes care during the past decade, achieving the HbA1c targets remains suboptimal among adults with diabetes [32]. Our data confirm the wide gap between the guidelines and the clinical practice in diabetes management in the Balkan region, with only $33.7 \%$ of patients at HbA1c goal according to IDF criteria (HbA1c at most $6.5 \%$ ) and $55.1 \%$ according to ADA criteria (HbA1c at most $7.0 \%$ ). Clinical inertia is recognized as a major factor that contributes to widespread failure to achieve evidence-based goals in patients with T2DM [33]. Even though more patients in our study were not adequately controlled, therapy was modified in only $28 \%$ of patients. Therefore, our results confirm that there are delays in treatment intensification in patients with suboptimal glycemic control in the Balkan region. Overall, introduction of a DPP-4 inhibitor was the most prevalent change when adding/switching to a different pharmacological class.

To the best of our knowledge the RECAP-DM Balkan study is the first study showing the incidence rate of side effects of SU therapy including hypoglycemia and weight gain in countries of the Balkan region. Its observational nature represents both a strength and a limitation. Potential for bias with non-randomized data collection is a well-known limitation of observational research. Nevertheless, the usefulness of observational studies is being increasingly recognized as an additional tool to inform current clinical practice and to guide healthcare decision-making. The extrapolation of RCT results into everyday practice may not be fully representative of a general diabetes population especially when it comes to incidence of hypoglycemia. The true incidence of hypoglycemia is often underestimated because RCTs of diabetes population usually exclude patients with severe episodes of hypoglycemia, recurrent hypoglycemic events, or hypoglycemia unawareness. Observational studies conducted under real-life conditions can provide valuable data regarding the use of antidiabetic drugs in everyday clinical practice as treatment is monitored in a real-world setting rather than under the rigorous constraint of an RCT. As a result of the subjective nature of the assessment, patient self-reported outcomes also have the potential for confounding. However, since many patients with diabetes may not always recognize or recall symptoms of hypoglycemia, or may have limited knowledge about hypoglycemia itself, it is possible that the true incidence of hypoglycemia was even higher than reported. Although this study tests prevalence of side effects and level of control achieved by patients using SUs, the results cannot be directly attributed to these drugs because of the lack of an adequate comparative group. As with any "real-life" results, the effectiveness (or lack thereof) and prevalence of side effects can be significantly influenced by the treatment patterns (the way patients are chosen to receive SUs).

\section{CONCLUSIONS}

Despite well-documented safety issues related to SU therapy such as hypoglycemia and weight gain, there are a large number of patients taking these agents in the Balkan region, either alone or as part of combination therapy. Although newer generation SUs are generally considered safe in terms of hypoglycemia, our data indicates that their use is associated with substantial risk of hypoglycemia. Furthermore, the results of our study provide evidence that the frequency and severity of hypoglycemia in SU-treated patients are associated with increased fear of hypoglycemia. Clinicians should be mindful of these findings when prescribing SUs and inform patients about the risk 
of hypoglycemia. Weight-friendly agents which carry a low risk of hypoglycemia such as incretin mimetics and SGLT-2 inhibitors represent a valuable therapeutic options that may help patients to achieve and sustain improved glycemic control and thus reduce diabetes-related complications in countries of the Balkan region.

\section{ACKNOWLEDGEMENTS}

Funding was provided by Merck Sharp \& Dohme d.o.o., Zagreb, Croatia; Merck Sharp \& Dohme, inovativna zdravila d.o.o., Ljubljana, Slovenia; Merck Sharp \& Dohme, Sofia, Bulgaria; MSD Idea, Inc., Belgrade, Serbia, for the research of this study. The study sponsors also funded article processing charges. Participating physicians/investigators were funded for their enrollment of patients. Merck Sharp \& Dohme d.o.o., Zagreb, Croatia funded the writer of the manuscript. Sanja Klobucar Majanovic wrote the initial and subsequent drafts of the manuscript after reviews and edits from coauthors. Andrej Janez, Ivaylo Lefterov, Sanja Tasic, and Tatjana Cikac reviewed and edited the manuscript. All authors take full responsibility for the work as a whole, including the study design and the decision to submit and publish the manuscript. All named authors meet the International Committee of Medical Journal Editors (ICMJE) criteria for authorship for this manuscript, take responsibility for the integrity of the work as a whole, and have given final approval for the version to be published. All authors had full access to all of the data in this study and take complete responsibility for the integrity of the data and accuracy of the data analysis. The authors would like to express their gratitude to all participating investigators for their commitment to this research effort. Rho Sigma Research \& Statistics performed the statistical analysis. Merck Sharp \& Dohme d.o.o., Zagreb, Croatia and Clinres farmacija d.o.o. designed and performed the study and collected and researched the data.

Disclosures. Sanja Klobucar Majanovic received funding from Merck Sharp \& Dohme for the enrollment of patients and received funding for the writing of the manuscript from Merck Sharpe and Dohme d.o.o., Zagreb, Croatia. Andrej Janez received funding from Merck Sharp \& Dohme for the enrollment of patients. Ivaylo Lefterov received funding from Merck Sharp \& Dohme for the enrollment of patients. Sanja Tasic received funding from Merck Sharp \& Dohme for the enrollment of patients. Tatjana Cikac received funding from Merck Sharp \& Dohme for the enrollment of patients.

Compliance with Ethics Guidelines. All procedures followed were in accordance with the ethical standards of the responsible committee on human experimentation (institutional and national) and with the Helsinki Declaration of 1964, as revised in 2013. Informed consent was obtained from all patients for being included in the study. This study was approved by the ethics committee in each participating country.

Data Availability. The datasets generated during and/or analyzed during the current study are available from the corresponding author on reasonable request.

Open Access. This article is distributed under the terms of the Creative Commons Attribution-NonCommercial 4.0 International License (http://creativecommons.org/licenses/ by-nc/4.0/), which permits any noncommercial use, distribution, and reproduction in any medium, provided you give appropriate credit to the original author(s) and the source, provide a link to the Creative Commons license, and indicate if changes were made.

\section{REFERENCES}

1. Barendse S, Singh H, Frier BM, Speight J. The impact of hypoglycaemia on quality of life and related patient-reported outcomes in type 2 diabetes: a narrative review. Diabet Med. 2012;29:293-302.

2. Eriksson JW, Bodegard J, Nathanson D, Thuresson M, Nystrom T, Norhammar A. Sulphonylurea 
compared to DPP-4 inhibitors in combination with metformin carries increased risk of severe hypoglycemia, cardiovascular events, and all-cause mortality. Diabetes Res Clin Pract. 2016;117:39-47.

3. Monami M, Dicembrini I, Kundisova L, Zannoni S, Nreu B, Mannucci E. A meta-analysis of the hypoglycaemic risk in randomized controlled trials with sulphonylureas in patients with type 2 diabetes. Diabetes Obes Metab. 2014;16:833-40.

4. Gautier JF, Monguillon P, Verier-Mine O, et al. Which oral antidiabetic drug to combine with metformin to minimize the risk of hypoglycemia when initiating basal insulin? A randomized controlled trial of a DPP4 inhibitor versus insulin secretagogues. Diabetes Res Clin Pract. 2016;116:26-8.

5. Gerstein HC, Miller ME, Byington RP, Action to Control Cardiovascular Risk in Diabetes Study Group, et al. Effects of intensive glucose lowering in type 2 diabetes. N Engl J Med. 2008;358:2545-59.

6. Cryer PE. Death during intensive glycemic therapy of diabetes: mechanisms and implications. Am J Med. 2011;124:993-6.

7. Zoungas S, Patel A, Chalmers J, et al. Severe hypoglycemia and risks of vascular events and death. N Engl J Med. 2010;363:1410-8.

8. Martín-Timón I, Cañizo-Gómez FJ. Mechanisms of hypoglycemia unawareness and implications in diabetic patients. World J Diabetes. 2015;6(7):912-26.

9. Kalra S, Mukherjee JJ, Venkataraman S, et al. Hypoglycemia: the neglected complication. Indian J Endocrinol Metab. 2013;17(5):819-34.

10. Roumie CL, Greevy RA, Grijalva CG, et al. Association between intensification of metformin treatment with insulin vs sulfonylureas and cardiovascular events and all-cause mortality among patients with diabetes. JAMA. 2014;311(22):2288-96.

11. Leiter LA, Yale J-F, Chiasson J-L, Harris SB, Kleinstiver P, Sauriol L. Assessment of the impact of fear of hypoglycemic episodes on glycemic and hypoglycemic management. Can J Diabetes. 2005;29:186-92.

12. Seaquist ER, Anderson J, Childs B, et al. Hypoglycemia and diabetes: a report of a workgroup of the American Diabetes Association and the Endocrine Society. Diabetes Care. 2013;36(5):1384-95.

13. Chan SP, Ji LN, Nitiyanant W, Baik SH, Sheu WH. Hypoglycemic symptoms in patients with type 2 diabetes in Asia-Pacific-real-life effectiveness and care patterns of diabetes management: the
RECAP-DM study. Diabetes Res Clin Pract. 2010;89(2):e30-2.

14. Alvarez Guisasola F, Tofé Povedano S, Krishnarajah G, Lyu R, Mavros P, Yin D. Hypoglycaemic symptoms, treatment satisfaction, adherence and their associations with glycaemic goal in patients with type 2 diabetes mellitus: findings from the Real-Life Effectiveness and Care Patterns of Diabetes Management (RECAP-DM) Study. Diabetes Obes Metab. 2008;10(Suppl 1):25-32.

15. Inkster B, Zammitt NN, Frier BM. Drug-induced hypoglycaemia in type 2 diabetes. Expert Opin Drug Saf. 2012;11(4):597-614.

16. Campbell IW. Comparing the actions of older and newer therapies on body weight: to what extent should these effects guide the selection of antidiabetic therapy? Int J Clin Pract. 2010;64:791-801.

17. Hirst JA, Farmer AJ, Dyar A, Lung TWC, Stevens RJ. Estimating the effect of sulfonylurea on HbA1c in diabetes: a systematic review and meta-analysis. Diabetologia. 2013;56:973-84.

18. Elliott L, Fidler C, Ditchfield A, Stissing T. Hypoglycemia event rates: a comparison between real-world data and randomized controlled trial populations in insulin-treated diabetes. Diabetes Ther. 2016;7(1):45-60.

19. Amiel SA, Dixon T, Mann R, Jameson K. Hypoglycaemia in type 2 diabetes. Diabet Med. 2008;25:245-54.

20. Cryer PE, Childs BP. Negotiating the barrier of hypoglycemia in diabetes. Diabetes Spectr. 2002; 15:20-7.

21. Cox DJ, Irvine A, Gonder-Frederick L, Nowacek G, Butterfield J. Fear of hypoglycemia: quantification, validation and utilization. Diabetes Care. 1987;10:617-21.

22. Gonder-Frederick LA, Schmidt KM, Vajda KA, et al. Psychometric properties of the Hypoglycemia Fear Survey-II for adults with type 1 diabetes. Diabetes Care. 2011;34(4):801-6.

23. Stargardt T, Gonder-Frederick L, Krobot KJ, Alexander CM. Fear of hypoglycaemia: defining a minimum clinically important difference in patients with type 2 diabetes. Health Qual Life Outcomes. 2009;7:91.

24. Wild D, von Maltzahn R, Brohan E, Christensen T, Clauson P, Gonder-Frederick L. A critical review of the literature on fear of hypoglycemia in diabetes: implications for diabetes management and patient education. Patient Educ Couns. 2007;68:10-5. 
25. Marrett E, Radican L, Davies MJ, Zhang Q. Assessment of severity and frequency of self-reported hypoglycemia on quality of life in patients with type 2 diabetes treated with oral antihyperglycemic agents: a survey study. BMC Res Notes. 2011;4:251.

26. Hajós TR, Polonsky WH, Pouwer F, Gonder-Frederick L, Snoek FJ. Toward defining a cutoff score for elevated fear of hypoglycemia on the Hypoglycemia Fear Survey Worry Subscale in patients with type 2 diabetes. Diabetes Care. 2014;37(1):102-8.

27. Schramm TK, Gislason GH, Vaag A, et al. Mortality and cardiovascular risk associated with different insulin secretagogues compared with metformin in type 2 diabetes, with or without a previous myocardial infarction: a nationwide study. Eur Heart J. 2011;32:1900-8.

28. Schopman JE, Simon AC, Hoefnagel SJ, Hoekstra JB, Scholten RJ, Holleman F. The incidence of mild and severe hypoglycaemia in patients with type 2 diabetes mellitus treated with sulfonylureas: a systematic review and meta-analysis. Diabetes Metab Res Rev. 2014;30:11-22.

29. Andersen SE, Christensen M. Hypoglycaemia when adding sulphonylurea to metformin: a systematic review and network meta-analysis. Br J Clin Pharmacol. 2016. doi:10.1111/bcp.13059.

30. van Dalem J, Brouwers MC, Stehouwer CD, et al. Risk of hypoglycaemia in users of sulphonylureas compared with metformin in relation to renal function and sulphonylurea metabolite group: population based cohort study. BMJ. 2016;13(354):i3625.

31. Inzucchi SE, Bergenstal RM, Buse JB, American Diabetes Association (ADA), European Association for the Study of Diabetes (EASD), et al. Management of hyperglycemia in type 2 diabetes: a patient-centered approach: position statement of the American Diabetes Association (ADA) and the European Association for the Study of Diabetes (EASD). Diabetes Care. 2012;35:1364-79.

32. Stark Casagrande S, Fradkin JE, Saydah SH, Rust KF, Cowie CC. The prevalence of meeting A1C, blood pressure, and LDL goals among people with diabetes, 1988-2010. Diabetes Care. 2013;36(8):22-1-2279.

33. Khunti K, Wolden ML, Thorsted BL, Andersen M, Davies MJ. Clinical inertia in people with type 2 diabetes: a retrospective cohort study of more than 80,000 people. Diabetes Care. 2013;36(11):3411-7. 